\title{
GYMNOPHALLUS REBECQUI n .sp. (syn. PARVATREMA sp. 1, J. REBEGQ, 1964) \\ (Digenea : Gymnophallidae) parasite intestinal d'Anatidés de Camargue (France)
}

\author{
P. BARTOLI*
}

RÉSUMÉ. Gymnophallus rebecqui n. sp. remplace Parvatrema sp. 1 J. Rebecq, 1964. Les métacercaires sont redécrites. Ce sont des parasites de Cerastoderma glaucum et de Abra ovata; ils se répartissent dans l'espace extrapalléal général de leurs hôtes. Les relations hôte-parasite sont étudiées et des altérations du manteau et de la coquille sont décrites chez les hôtes parasités. Les stades adultes correspondants ont été obtenus expérimentalement chez divers Anatidés d'élevage et chez Larus argentatus michaellis. Dans la nature, les stades ovigères ont été trouvés dans l'intestin antérieur et moyen de Aythya ferina, A. fuligula et Anas clypeata. Les adultes sont décrits et comparés à plusieurs espèces proches de la famille des Gymnophallidae. L'étude anatomique des métacercaires et surtout celle des adultes montre que cette espèce n'appartient pas au genre Parvatrema mais possède tous les caractères du genre Gymnophallus.

\section{Gymnophallus rebecqui n. sp. (syn. Parvatrema sp. 1, J. Rebecq, 1964) (Digenea : Gymnophallidae), an intestinal parasite of Ducks from Camargue (France).}

SUMMARY. Gymnophallus rebecqui n. sp. replace Parvatrema sp. 1 J. Rebecq, 1964. Metacercariae are described. They occur free in the extrapallial space of Cerastoderma glaucum and Abra ovata, at the central part of the valves. Pallial epithelium hypertrophy is induced by metacercariae and damages are produced at the inner face of the shell of Abra ovata. Adults have been reared in laboratory hosts (Aythya ferina, A. fuligula, Anas platyrhynchos, Tadorna tadorna and Larus argentatus michaellis). Natural adults have been discovered in the anterior and median gut of Aythya ferina, A. fuligula and Anas clypeata. Adults are described and compared with other related Gymnophallid species. This new species is not a member of Parvatrema but belongs to Gymnophallus genera.

Les Gymnophallidae sont des Trématodes dont le cycle biologique hétéroxène se déroule en milieu marin ou saumâtre. Les premiers hôtes intermédiaires sont des Mollusques Lamellibranches ou Prosobranches; les deuxièmes hôtes sont habituel-

* Laboratoire de Zoologie mari ıe, Faculté des Sciences et Techniques de Saint-Jérôme, Université d'Aix-Marseille III, F 13397 Marseille Cedex 13.

Accepté le 9 novembre 1982. 
lement des Bivalves, plus rarement des Prosobranches ou des Annélides Polychètes ; les hôtes définitifs sont des Oiseaux, soit des Laro-limicoles, soit des Anatidés.

Les Gymnophallidae sont très bien représentés en Camargue; nous en avons dénombré huit espèces (P. Bartoli, 1974). Une telle richesse est liée au fait que dans cette région abondent non seulement les Mollusques et les Annélides mais aussi les Oiseaux hôtes définitifs de ces parasites. En effet, pour plusieurs de ces Oiseaux, la Camargue est une étape sur les voies migratoires ; pour d'autres, comme les Anatidés, elle représente l'aire d'hivernage ; pour d'autres enfin, la Camargue est l'aire de reproduction. Tout au long de l'année, cette région voit donc séjourner, pendant des périodes de temps plus ou moins longues, une grande variété d'Oiseaux hôtes définitifs de Gymnophallidae.

Dans la partie saumâtre de la Camargue, nous avons retrouvé en abondance une espèce qui avait été seulement signalée par J. Rebecq (1964) sous le nom de Parvatrema sp. 1. C'est cette espèce que nous redécrivons ici sous le nom de Gymnophallus rebecqui n. sp. Grâce aux contaminations artificielles, nous avons obtenu la forme adulte correspondante chez plusieurs Oiseaux; les adultes naturels ont été trouvés chez divers Anatidés de Camargue.

\section{I - Les métacercaires}

\section{1 - Les deuxièmes hôtes intermédiaires}

A l'inverse de la plupart des Gymnophallides de Camargue, Gymnophallus rebecqui $\mathrm{n}$. sp. admet seulement deux deuxièmes hôtes intermédiaires : Cerastoderma glaucum Poiret et Abra ovata (Philippi).

\section{2 - Description}

Les métacercaires, non enkystées, ont la même forme et les mêmes dimensions, quelle que soit l'espèce du Mollusque deuxième hôte qui les abrite. La description qui suit résulte de l'examen d'un très grand nombre de métacercaires.

\section{Dimensions}

Les dimensions, en $\mu \mathrm{m}$, sont celles de 40 individus montés in toto.

Longueur du corps : 132-202 (168).

Largeur du corps : 105-142 (122).

Ventouse orale : $30-35(32) \times 32-43(38,5)$.

Ventouse ventrale : 25-30 (27) $\times$ 28-33 (30).

Rapport ventousaire : $1,11-1,36(1,18)$.

Espace préacétabulaire : 66-108 (86).

Espace postacétabulaire : 37-72 (56).

Pharynx : 20-26 (24) × 25-33 (27).

Rapport ventouse orale-pharynx, VO/P : 1,20-1,52 (1,32).

Testicules : 25-35 (31) × 15-28 (19).

Ovaire : $19-28(24) \times 13-17(15)$. 


\section{Tégument}

Le corps est revêtu d'épines dirigées vers l'extrémité postérieure ; elles sont disposées en quinconce. Elles sont à peine plus nombreuses dans la moitié antérieure du corps que dans sa moitié postérieure.

Certaines surfaces du corps sont dépourvues d'épines ( $f i g .1$ ). Sur la face ventrale du corps, les aires glabres offrent l'aspect d'un Y dont les branches antérieures s'étendent de part et d'autre de la ventouse ventrale; le tronc impair s'étale entre la ventouse ventrale et l'extrémité postérieure du corps. Immédiatement en avant de l'orifice génital, une petite surface réniforme est aussi dépourvue d'épines; celle-ci est particulièrement visible lorsque les métacercaires sont en élongation.
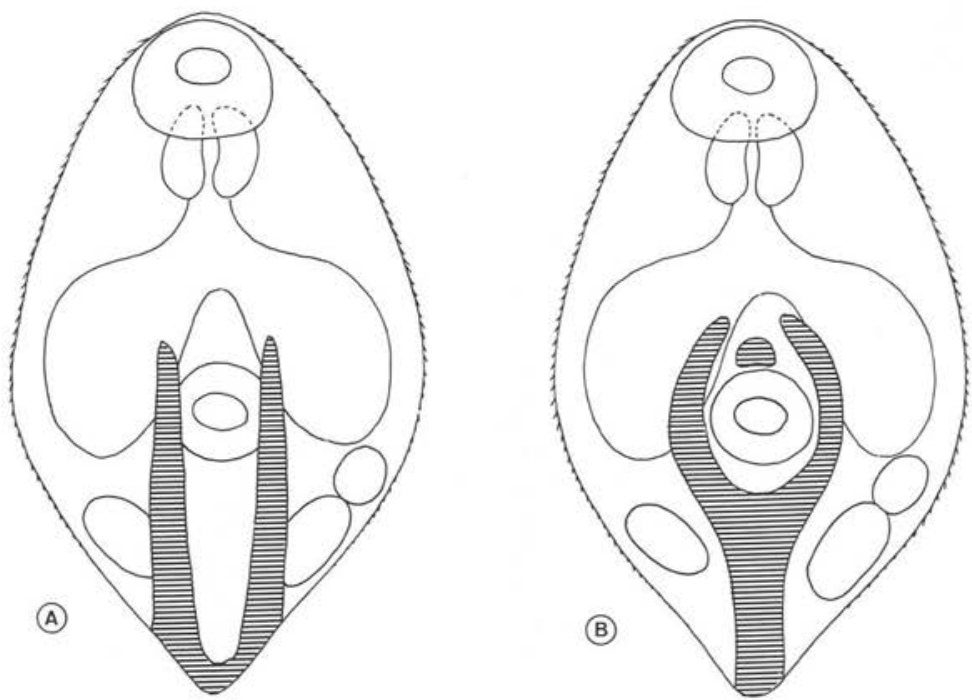

FIG. I. - Gymnophallus rebecqui n. sp. Métacercaires parasites de Cerastoderma glaucum et A bra ovata. Les aires tégumentaires dépourvues d'épines sont hachurées. A. : face dorsale ; B : face ventrale.

Sur la face dorsale du corps, les surfaces non spinulées se présentent sous l'aspect de deux bandes parallèles entre elles; elles s'étendent de part et d'autre de la ligne médiodorsale, depuis le niveau du bord antérieur de la ventouse ventrale jusqu'à l'extrémité postérieure du corps ; là, ces deux bandes fusionnent entre elles et avec l'aire glabre de la face ventrale.

Les épines ont des dimensions assez importantes; les plus volumineuses, celles de la région médio-dorsale, atteignent une longueur de $2,5 \mu \mathrm{m}$. Leur extrémité libre n'est jamais simple; on observe toujours au-moins deux mucrons très acérés dans les épines de la région médio-ventrale; on en compte trois et même quatre dans celles de la région médio-dorsale (fig. 2).

\section{Glandes céphaliques}

Les glandes céphaliques sont nombreuses ( fig. 3); on peut difficilement en évaluer le nombre exact. De chaque côté, elles se répartissent généralement en deux ensembles bien distincts; l'un est disposé dans la région postéro-latérale de la ventouse orale ; 


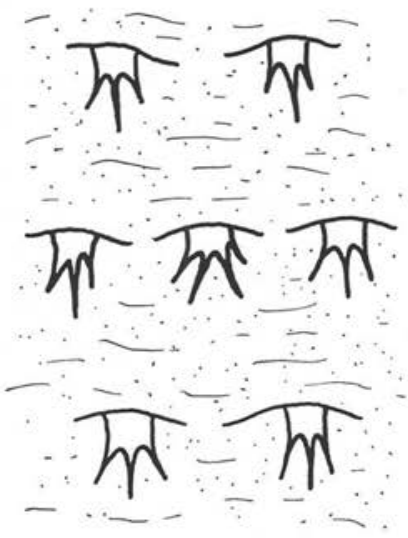

FIG. 2. - Gymnophallus rebecqui n. sp. Métacercaire. Épines tégumentaires de la face dorsale.

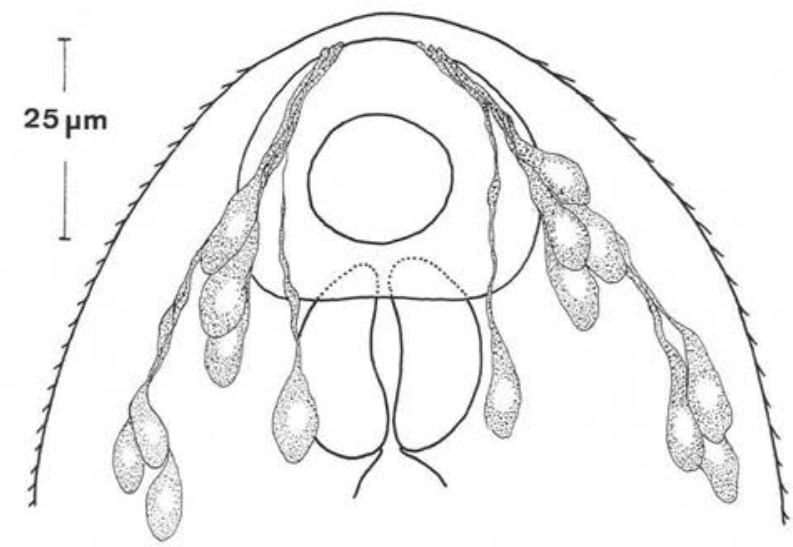

FIG. 3. - Gymnophallus rebecqui n. sp. Métacercaire. Glandes céphaliques (vue dorsale).

l'autre est localisé près du bord du corps, au niveau de l'extrémité postérieure du pharynx. Parfois, on observe de part et d'autre du pharynx ou au-dessus de celui-ci, le corps cellulaire d'une ou deux autres glandes céphaliques. Les canalicules, mal visibles, s'abouchent sur le bord antérieur de l'orifice buccal.

\section{Ventouses}

La ventouse orale est sensiblement plus large que longue.

La ventouse ventrale est aussi légèrement plus large que longue; les six terminaisons sensorielles du cycle acétabulaire externe sont parfaitement bien distinctes.

\section{Appareil digestif}

La bouche s'ouvre au centre de la ventouse orale. Le prépharynx est absent. Le pharynx est ovoïde; ses dimensions sont légèrement inférieures à celles de la ventouse ventrale. 
L'œsophage est court. Les caecums digestifs sont extrêmement volumineux; leur limite postérieure atteint généralement le niveau du centre de la ventouse ventrale, plus rarement le niveau de son bord postérieur.

\section{Appareil génital mâle}

L'appareil génital des métacercaires est presque aussi développé que celui des adultes.

Les testicules, ovoïdes, sont placés près de la paroi du corps ; ils sont entièrement contenus dans l'espace postacétabulaire (fig. 4). Les spermiductes sont très nettement visibles; ils s'échappent du milieu de la longueur des testicules. La vésicule séminale est très distinctement divisée transversalement (fig. 5). La pars prostatica a les mêmes dimensions que chez l'adulte. Les cellules prostatiques sont énormément développées; elles remplissent tout l'espace intercaecal. Le canal éjaculateur peut être observé très facilement.

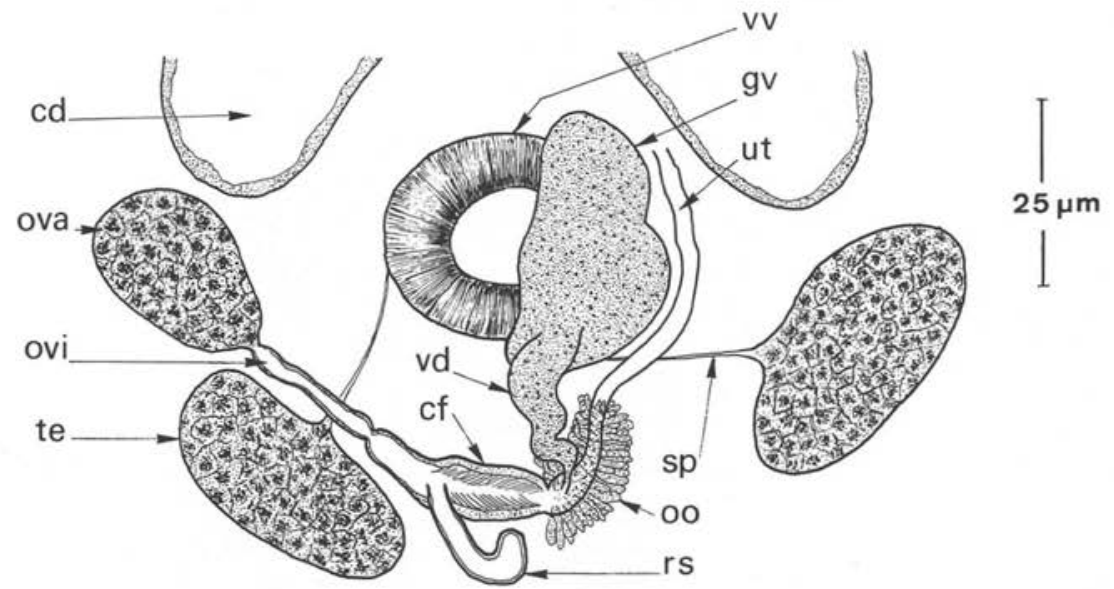

FIG. 4. - Gymnophallus rebecqui n. sp. Métacercaire.

Région proximale des appareils génitaux mâle et femelle (vue dorsale).

cd : caecum digestif ; $\mathrm{cf}$ : chambre de fécondation ciliée; gv : glande vitelline; oo : ootype ; ova : ovaire ; ovi : oviducte ; rs : réceptacle séminal; sp : spermiducte ; te : testicule ; ut : utérus ; vd : vitelloducte; vv : ventouse ventrale.

\section{Appareil génital femelle}

L'ovaire est ovoïde; ses dimensions sont inférieures à celles des testicules. Il est disposé au niveau de la ventouse ventrale, tantôt à droite, tantôt à gauche; il entre en contact avec la face antérieure de l'un des deux testicules. La chambre de fécondation, ciliée, reçoit à son extrémité proximale le réceptacle séminal et à son extrémité distale le vitelloducte (fig. 4). Le canal de Laurer est absent. Les follicule vitellins sont groupés en une masse unique plus ou moins lobée. L'ootype est très nettement visible. L'utérus rejoint l'espace préacétabulaire en passant entre la ventouse ventrale et le testicule opposé à l'ovaire ; il décrit une boucle plus ou moins importante qui s'étend parfois jusque sur la ventouse orale. L'utérus débouche dans l'atrium génital.

\section{Atrium génital}

L'atrium génital est très court (fig. 5); il s'ouvre à l'extérieur par le pore génital. Celui-ci est extrêmement petit ; il est disposé au contact de la ventouse ventrale, sur son 


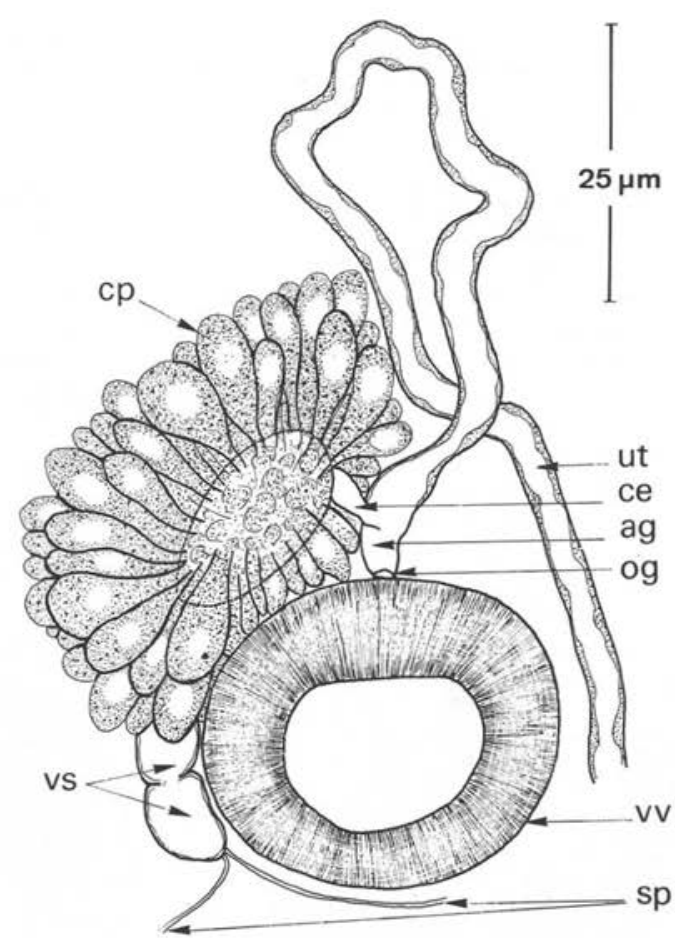

FIG. 5. - Gymnophallus rebecqui $\mathrm{n}$. sp. Métacercaire.

Région distale des appareils génitaux mâle et femelle (vue ventrale).

ag : atrium génital; ce : canal éjaculateur ; $\mathrm{cp}$ : cellules prostatiques enveloppant la pars prostatica ; og : orifice génital ; sp : spermiductes; ut : utérus ; vs : vésicule séminale bipartie ; vv : ventouse ventrale.

bord antérieur, en position médiane. L'orifice génital n'est pas entouré de fibres musculaires ; on n'observe aucune dépression de la paroi du corps le long de la ligne médioventrale, en avant de l'orifice génital.

\section{Appareil excréteur}

Les cellules à flamme vibratile sont disposées les unes par rapport aux autres selon la formule : $2[(2+2)+(2+2)]=16$.

Les quatre protonéphridies du premier groupe sont réparties dans l'espace préacétabulaire, celles du second sont disposées au niveau de la ventouse ventrale et en arrière de celle-ci ( fig. 6). De chaque côté, le canal collecteur principal, cilié, se jette à l'extrémité d'une branche de la vessie. La vessie est constituée par un tronc impair peu étendu, plus ou moins boursouflé et par deux branches latérales, chacune d'elles s'insinuant entre le testicule et l'extrémité d'un caecum digestif. L'extension des branches vésicales vers la région antérieure est peu importante; elles n'embrassent pas les caecums. Chacune de ces branches présente, près du lieu où elles se jettent dans le tronc, un diverticule souvent très accusé. Les granulations excrétrices ont un diamètre inférieur à $2,5 \mu \mathrm{m}$; elles ne sont pas nombreuses, aussi la couleur générale de la métacercaire demeure-t-elle très claire. Le pore excréteur est terminal. 


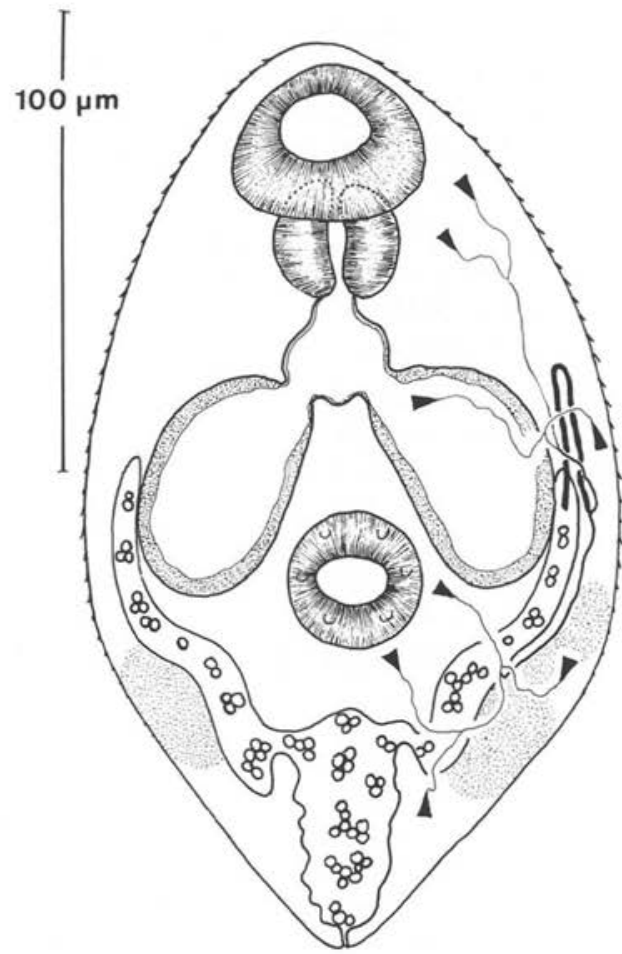

Fig. 6. - Gymnophallus rebecqui n. sp. Métacercaire. Appareil excréteur.

\section{3 - Microhabitat}

Lorsque les métacercaires de Gymnophallides parasitent des Lamellibranches, elles se répartissent habituellement entre la coquille et le manteau de leur hôte. Pour une espèce donnée de Gymnophallide, les métacercaires occupent une fraction déterminée de l'espace extrapalléal (P. Bartoli, 1974). Ainsi, chez Cerastoderma glaucum, les métacercaires de Gymnophallus rebecqui n. sp. se localisent principalement dans l'espace extrapalléal général; chez Abra ovata, on les rencontre à la fois dans l'espace extrapalléal général et dans l'espace extrapalléal sous-articulaire.

\section{Relations hôte-parasite.}

Les relations hôte-parasite ont été étudiées plus particulièrement dans le cas du deuxième hôte intermédiaire Abra ovata.

Sur l'ensemble des métacercaires, une très faible proportion mène une existence solitaire. Elles ne sont pas alors localisées en un point précis de l'espace extrapalléal général mais sont réparties un peu partout dans ce microhabitat. 
Cependant, dans l'immense majorité des cas, les métacercaires se regroupent pour former des agrégats plus ou moins importants. De tels rassemblements sont toujours visibles dans la région dorsale de l'espace extrapalléal général de chaque valve ; on peut aussi en rencontrer en d'autres points dans ces deux microhabitats. Dans la région de ces agrégats de métacercaires, la conformation du manteau est fortement modifiée ( fig. 7). Les coupes histologiques révèlent un épithélium palléal considérablement épaissi et très chromophile. Des proliférations en forme de villosités apparaissent ; toutefois, elles n'entourent jamais les parasites. Les métacercaires sont en contact par leur face ventrale avec le manteau, par leur face dorsale avec la coquille. Du côté interne de cette dernière, des dégradations apparaissent en regard de la région où les métacercaires sont rassemblées. Ces lésions, en forme de cupule, sont creusées dans l'épaisseur du test ; elles correspondent aux épaississements palléaux.

\section{II - Développement expérimental des métacercaires}

\section{1 - Développement in vivo.}

L'appareil génital des métacercaires de Gymnophallus rebecqui n. sp. est très développé. Une simple élévation de la température du milieu de vie suffit à déclancher l'activité génitale. La culture in vitro des métacercaires dans un milieu de Ringer additionné d'antibiotiques maintenu en étuve à $40^{\circ} \mathrm{C}$ permet d'observer une protandrie. Des spermatozoïdes actifs apparaissent dans les testicules au bout de 18 heures; ils remplissent et distendent la vésicule séminale 40 heures après le début de l'expérimentation.

\section{2 - Développement chez les Oiseaux.}

Plusieurs Oiseaux ont été contaminés avec les métacercaires de Gymnophallus rebecqui $\mathrm{n}$. sp. Les vers adultes ont été obtenus chez les hôtes expérimentaux suivants :

Aythya ferina (L.) : Canard Milouin.

Aythya fuligula (L.) : Canard Morillon.

Anas platyrhynchos: Canard Colvert.

Tadorna tadorna (L.) : Tadorne de Belon.

Larus argentatus michaellis (Naumann) : Goéland argenté à pieds jaunes.

Les repas infestants étaient composés soit de Cerastoderma glaucum, soit de Abra ovata. Dans tous les cas, nous avons recueilli dans l'intestin antérieur et dans l'intestin moyen de ces Oiseaux, un grand nombre de vers adultes, tous semblables entre eux (fig. 8). 

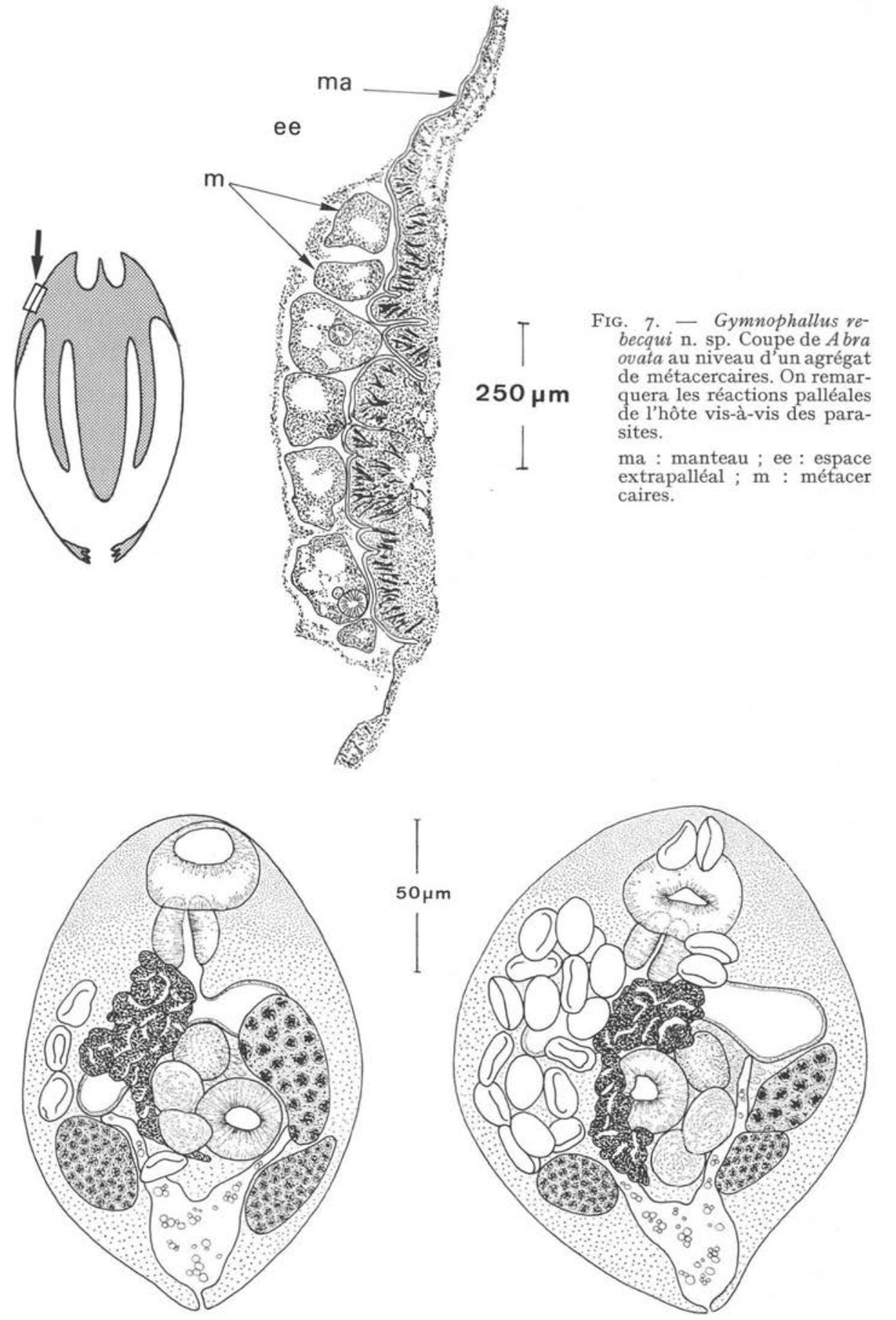

FIG. 8. - Gymnophallus rebecqui n. sp. Adultes expérimentaux. Intestin antérieur de Larus argentatus michaellis (hôte expérimental). 


\section{III — Les adultes naturels}

\section{1 - Hôtes définitifs naturels et prévalence.}

Aythya ferina : 8 exemplaires parasités sur 9 individus examinés.

Aythya fuligula : 3 exemplaires contaminés sur 5.

Anas clypeata : 1 exemplaire parasité sur deux.

\section{2 - Abondance.}

Un Aythya ferina abattu en Camargue était parasité par approximativement 8000 vers adultes.

\section{3 - Microhabitat.}

Les adultes naturels de Gymnophallus rebecqui n. sp. se répartissent le long de l'intestin antérieur et moyen. Les vers ne vivent pas attachés aux villosités mais dans l'épaisseur du film muqueux qui tapisse la paroi intestinale. Ils sont ainsi progressivement entraînés vers l'aval avec le chyle intestinal. De nombreux individus morts se rencontrent dans l'intestin postérieur et sont éliminés avec les déjections dans le milieu extérieur.

\section{4 - Longévité.}

La vie des Gymnophallides adultes, parasites de l'intestin, est de courte durée. Les infestations expérimentales de Larus argentatus montrent qu'elle ne dépasse pas cinq jours. Cette brièveté n'est pas liée à la nature expérimentale de l'hôte. En effet, les contaminations artificielles, par les métacercaires de Gymnophallus rebecqui n. sp., d'hôtes définitifs naturels tels que Aythya ferina ou A. fuligula montrent que la durée de vie des adultes ne dépasse pas six jours.

\section{5 - Description.}

La description qui suit repose sur l'examen de cinquante individus, vivants ou montés en préparation, recueillis chez Aythya ferina et A. fuligula (fig. 9).

\section{Dimensions}

Les dimensions, en $\mu \mathrm{m}$, sont celles de 20 exemplaires recueillis dans l'intestin antérieur de Aythya ferina.

Longueur du corps : 132-240 (186).

Largeur du corps : 124-165 (138).

Ventouse orale : $32-42(36) \times 37-48(42)$

Ventouse ventrale : 25-34 $(31,5) \times 30-38(33)$.

Rapport ventousaire : $1,00-1,32(1,15)$.

Espace préacétabulaire : 71-135 (103).

Espace postacétabulaire : 44-83 (58).

Pharynx : 20-26 (23) × 19-28 (22).

Rapport ventouse orale-pharynx, VO/P : 1,36-1,86 $(1,58)$. 

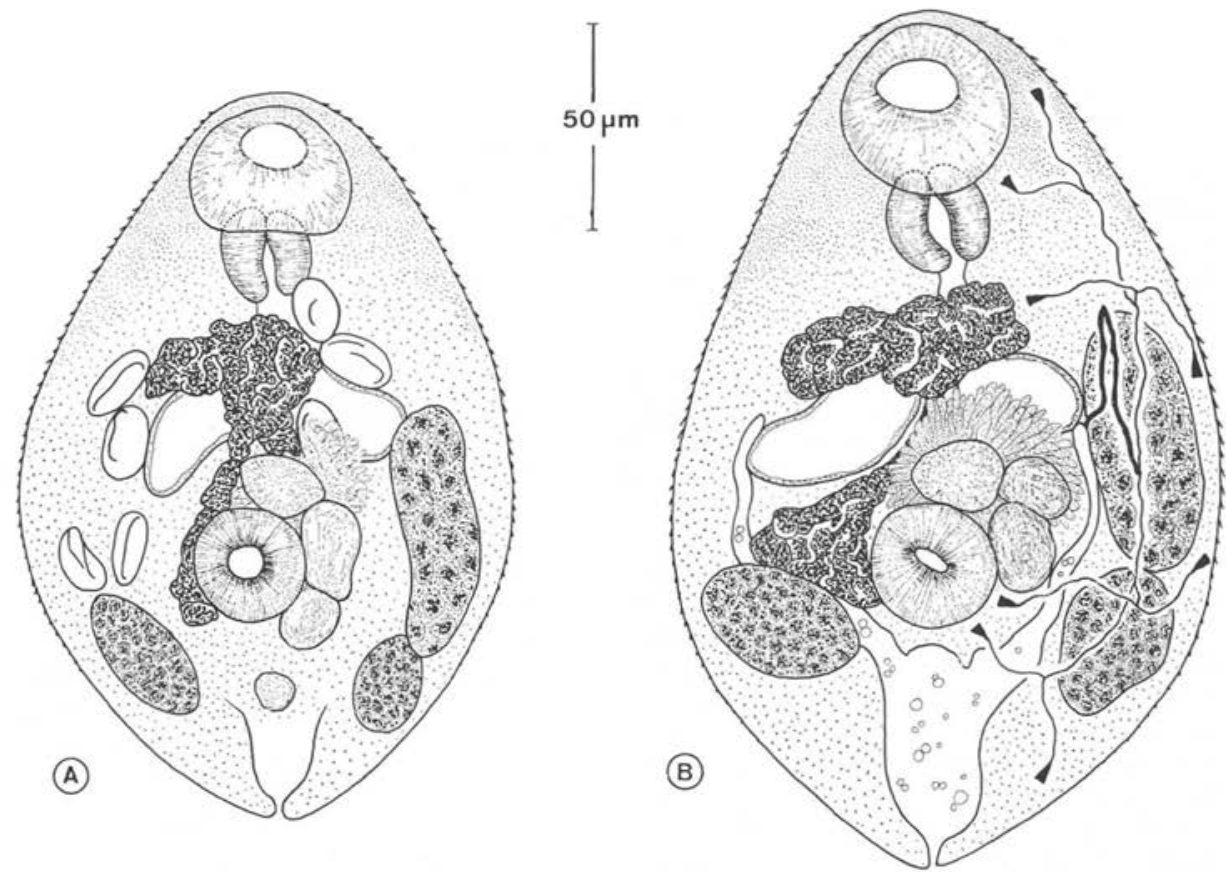

FIG. 9. - Gymnophallus rebecqui n. sp. Intestin antérieur de Aythya ferina (Camargue). A : individu ovigère (vue ventrale) ; B : postmétacercaire (appareils génital et excréteur) (vue ventrale).

Testicules : 28-43 (34) $\times$ 15-31 (23).

Vésicule séminale : $22-40(30) \times 12-22(17)$.

Partie prostatique : $19-40(30) \times 12-25(19)$.

Ovaire : $37-62(49) \times 22-31(26)$.

Eufs : $17-19(18) \times 8-12(10)$.

\section{Tégument}

Les épines sont assez volumineuses, disposées en quinconce et dirigées vers l'arrière du corps. Elles sont un peu plus nombreuses dans la région antérieure du corps; leur taille augmente légèrement à mesure que l'on se rapproche de l'extrémité postérieure.

\section{Ventouses}

Le plus souvent, la ventouse orale est très nettement subterminale. Toujours légèrement aplatie, elle ne possède aucun diverticule en forme de corne.

La ventouse ventrale, presque circulaire, est située approximativement à la limite des tiers moyen et postérieur.

\section{Appareil digestif}

La bouche s'ouvre au centre de la ventouse orale. Le prépharynx est absent. Le pharynx est globuleux. L'œsophage, généralement bien visible, conduit dans deux caecums digestifs divergents; leur limite postérieure atteint souvent mais pas toujours, le bord antérieur de la ventouse ventrale. 


\section{Appareil génitale mâle}

Généralement les testicules ont un aspect ovoïde ; toutefois, leurs contours ne sont pas toujours parfaitement réguliers. Ils sont situés entièrement en arrière de la ventouse ventrale, leur grand axe étant le plus souvent parallèle à la paroi du corps. Les spermiductes sont toujours très difficiles à mettre en évidence; ils conduisent dans une vésicule séminale bipartite dont la longueur correspond approximativement à celle de la ventouse ventrale. La division transversale de la vésicule séminale est toujours très nettement visible, que les exemplaires soient vivants ou fixés. Généralement, la vésicule séminale est disposée du côté de l'ovaire, entre celui-ci et la ventouse ventrale. La pars prostatica est une chambre volumineuse, de forme ovoïde, plus rarement sphérique ; elle est disposée près du bord antérieur de la ventouse ventrale. Les cellules prostatiques sont extraordinairement bien développées et sont particulièrement bien distinctes sur les individus vivants ; elles demeurent encore visibles sur les exemplaires fixés et montés en préparations. Le canal éjaculateur est court; aucune cellule glandaire ne lui est associée.

\section{Appareil génital femelle}

L'ovaire est énorme ; ses dimensions sont très largement supérieures à celles des testicules. Il est disposé tantôt à gauche, tantôt à droite, en avant et au contact de l'un des deux testicules. L'oviducte est relativement long. La chambre de fécondation est ciliée dans sa partie postérieure ; elle reçoit un réceptacle séminal toujours distinct, bourré de spermatozoïdes. Le canal de Laurer est absent. Les glandes vitellines sont constituées par des follicules groupés en une seule masse très lobulée. Celle-ci est disposée en avant de la ventouse ventrale mais jamais au niveau de celle-ci. Le vitelloducte offre rarement l'aspect d'un conduit; il est souvent distendu par l'accumulation de granules vitellins formant ainsi une masse disposée à la suite des follicules. L'ootype est bien visible. L'utérus rejoint la région préacétabulaire en s'insinuant entre la ventouse ventrale et le testicule opposé à l'ovaire ; il y décrit plusieurs circonvolutions puis vient déboucher dans l'atrium génital. Les œufs sont operculés et embryonnés; leur nombre n'est jamais très élevé, 47 en moyenne.

TABleau I. - Dimensions comparées des adultes de Gymnophallus rebecqui n. sp. recueillis dans Aythya ferina (infestation naturelle) et de ceux obtenus expérimentalement dans Larus argentatus michaellis autopsié deux jours après sa contamination.

\begin{tabular}{lcc}
\hline & $\begin{array}{c}\text { Aythya ferina } \\
\text { (hôte naturel) }\end{array}$ & $\begin{array}{c}\text { Larus argentatus } \\
\text { (hôte expérimental) }\end{array}$ \\
\hline Longueur & $132-240(186)$ & $136-223(174)$ \\
Largeur & $124-165(138)$ & $83-136(108)$ \\
Ventouse orale & $32-42(36) \times 37-48(42)$ & $28-37(33) \times 28-42(36)$ \\
Ventouse ventrale & $25-34(31,5) \times 30-38(33)$ & $25-31(28) \times 25-32(29)$ \\
Pharynx & $20-26(23) \times 19-28(22)$ & $19-25(22) \times 15-24(20)$ \\
Testicule opposé à l'ovaire & $25-43(35) \times 19-25(21)$ & $30-45(38) \times 15-20(19)$ \\
Ovaire & $37-62(49) \times 22-31(26)$ & $45-60(50) \times 15-30(22)$ \\
Eufs & $17-19(18) \times 8-12(10)$ & $17-20(19) \times 7-12(10)$ \\
VO/VV & $1-1,32(1,15)$ & $1-1,28(1,17)$ \\
VO/P & $1,36-1,86(1,58)$ & $1,30-1,85(1,50)$ \\
\hline
\end{tabular}




\section{Atrium génital}

Il est extrêmement court et débouche à l'extérieur par le pore génital. Celui-ci, médio-ventral, est situé sur le bord antérieur de la ventouse ventrale ; il est extrêmement petit. Aucune cellule glandulaire ne se jette dans l'atrium génital. Le pore génital n'est pas entouré de fibres musculaires; on n'observe pas de dépression préacétabulaire.

\section{Appareil excréteur}

Les protonéphridies sont groupées entre elles selon la formule : $2[(2+2)+$ $(2+2)]=16$. Lorsque les individus sont immatures, la vessie excrétrice renferme encore quelques granulations. Les branches vésicales sont généralement grêles; chacune émet un diverticule en arrière de la ventouse ventrale. Le tronc impair est peu étendu; il communique avec l'extérieur par un pore excréteur terminal.

Les vers adultes recueillis chez Aythya ferina, A. fuligula ou Anas clypeata infestés naturellement sont identiques à ceux obtenus chez les divers hôtes expérimentaux (tableau I). On peut donc affirmer que la nature expérimentale de l'hôte définitif ne modifie pas les caractères morphologiques et anatomiques des adultes.

\section{IV - Discussion}

Nous dédions cette espèce à $\mathrm{J}$. Rebecq qui a été le premier a en trouver les métacercaires (1964). Celles-ci, localisées " au niveau du manteau " de Cerastoderma glaucum, ont été décrites et appelées par cet auteur Parvatrema sp. 1.

Nous nous sommes rendu sur le lieu même où J. Rebecq avait récolté les $C$. glaucum infestés (Étang du Fournelet; station XIX de J. Rebecq). Nous avons trouvé dans ces Lamellibranches de nombreuses métacercaires appartenant à une seule et même espèce que nous avons aussitôt identifiée comme Parvatrena sp. $1 \mathrm{~J}$. Rebecq, 1964.

Nous avons noté quelques différences avec la description originale de cette métacercaire, la plus importante étant celle relative à la formule excrétrice. J. Rebecq donne : $2[(2+2)+2]=12$ alors que, chez la métacercaire comme chez l'adulte, nous avons toujours trouvé $2[(2+2)+(2+2)]=16$.

Or J. Rebecq (1964) voyait dans la formule excrétrice : $2[(2+2)+2]=12$, un caractère générique ; c'est pourquoi il attribua cette espèce au genre Parvatrema. En 1967, E. A. Bowers et B. L. James ont montré que le genre Parvatrema n'était pas seulement caractérisé par une formule excrétrice tronquée mais aussi par la formule complète $2[(2+2)+(2+2)]=16$. Par ailleurs, Parvatrema sp. 1 ne possède aucun des autres caractères du genre Parvatrema tel qu'il a été défini par R. M. Cable (1953) puis émendé par B. L. James (1964) ; par contre, cette espèce présente tous les caractères du genre Gymnophallus.

Gymnophallus rebecqui n. sp. ressemble à Gymnophallus gibberosus B. Loos Frank, 1971, à Parvatrema isostoma M. M. Belopolskaja, 1966, à Gymnophallus nereicola J. Rebecq et G. Prevot, 1962, à Meiogymnophalhus minutus (T. S. Cobbold, 1859) et à Gymnophallus fossarum P. Bartoli, 1965. 


\section{1 - Comparaison avec Gymnophallus gibberosus.}

Au stade adulte, G. gibberosus est un parasite des caecums intestinaux et du rectum d'Anatidés ; sa formule excrétrice est complexe : $2[(2+2+2)+(2+2+2)]=$ 24. En dehors de ces caractères, les diverses mensurations éloignent encore ces deux espèces l'une de l'autre.

Au stade métacercaire, ces deux Gymnophallides parasitent des Mollusques voisins : Cerastoderma edule pour G. gibberosus, C. glaucum pour G. rebecqui n. sp. Toutefois, les formules excrétrices sont radicalement différentes.

\section{2 - Comparaison avec Parvatrema isostoma.}

$\mathrm{Au}$ stade adulte, Parvatrema isostoma est un parasite de Charadriiformes. MM. Belopolskaja (1966) décrit chez cette espèce des cellules prostatiques débouchant directement dans l'atrium génital. Par ailleurs, l'illustration montre une vésicule séminale de structure unique, non bipartite. Tous ces caractères éloignent $P$. isostoma de Gymnophallus rebecqui n. sp.

Les métacercaires de Gymnophallus rebecqui n. sp. ressemblent extrêmement aux métacercaires attribuées à Parvatrema isostoma par A. V. Dolghikh (1968) et que cet auteur signale chez Cerastoderma edule et Abra ovata de la Mer Noire. Malheureusement, ces formes larvaires ont été rattachées à $P$. isostoma en vertu du seul caractère de la ressemblance morphologique; la filiation métacercaire-adulte n'a pas été démontrée expérimentalement. Nous pensons que les métacercaires parasites des $C$. glaucum et $A$. ovata de la Mer Noire pourraient correspondre à G. rebecqui n. sp.

\section{3 - Comparaison avec Gymnophallus nereicola.}

Gymnophallus nereicola est un parasite intestinal de Charadriiformes (P. Bartoli, 1972) ; l'expérimentation montre que le développement des métacercaires est impossible chez les Anatidés. Chez G. nereicola, le deuxième hôte intermédiaire n'est pas un Mollusque mais une Annélide polychète : Nereis diversicolor. En dehors de ces caractères liés au cycle de vie, G. rebecqui n. sp. et $G$. nereicola diffèrent par leurs mensurations et par le développement des cellules prostatiques.

\section{4 - Comparaison avec Meiogymnophallus minutus et Gymnophallus fossarum.}

Meiogymnophallus minutus et Gymnophallus fossarum sont extrêmement proches, tant par leur cycle de vie que par leurs diverses dimensions (A. Sannia, 1977 ; P. Bartoli, 1972). Ces deux espèces parasitent des Charadriiformes. Les vers adultes diffèrent de ceux de Gymnophallus rebecqui n. sp. par leurs dimensions, le développement des cellules prostatiques, la forme et l'extension des glandes vitellines.

Les métacercaires de Gymnophallus rebecqui n. sp. s'éloignent des métacercaires de $M$. minutus et de celles de $G$. fossarum par les dimensions du pharynx et par l'importance et la répartition des épines tégumentaires. Les métacercaires de 
M. minutus parasitent Cerastoderma edule, celles de G. fossarum se rencontrent chez C. glaucum ; chez ces deux Gymnophallides, le microhabitat est limité à la périphérie de l'espace extrapalléal tandis qu'il est réduit au centre des valves de $C$. glaucum pour Gymnophallus rebecqui n. sp.

L'endémiotope de Gymnophallus rebecqui se situe dans les aires saumâtres de la Camargue et correspond aux étangs fermés, sans communication avec la mer, ou aux fonds lagunaires euryhalins. C'est là que prochainement se porteront nos efforts en vue d'identifier le premier hôte intermédiaire et de découvrir les cercaires de ce Gymnophallide.

\section{BIBLIOGRAPHIE}

Bartoli P. : Les cycles biologiques de Gymnophallus nereicola J. Rebecq et G. Prévot, I962 et de $G$. fossarum P. Bartoli, 1965, espèces jumelles parasites d'Oiseaux de rivages marins (Trematoda, Digenea, Gymnophallidae). Ann. Parasitol. Hum. Comp., 1972, 47, 193-223.

Bartoli P. : Recherches sur les Gymnophallidae F. N. Morozov, 1955 (Digenea), parasites d'Oiseaux des côtes de Camargue : systématique, biologie et écologie. Thèse Université AixMarseille, 1974, no CNRS AO $9429,33^{8} \mathrm{p}$.

Belopolskaja M. M. : Parvatrema isostoma n. sp. des Charadriiformes des côtes de la Mer Noire (en russe). Vest. Leningr. Univ. Ser. biol., I966, 21, 147-148.

Bowers E. A., James B. L. : Studies on the morphology, ecology and life history of Meiogymnophallus minutus (Cobbold, I859) comb. nov. (Trematoda : Gymnophallidae). Parasitology, G.B., 1967, 57, 281-300.

CABLE R. M. : The life cycle of Parvatrema borinqueñae gen. et sp. nov. (Trematoda : Digenea) and the systematic position of the subfamily Gymnophallinae. J. Parasitol., $1953,39,408-421$.

DolghikH A. V. : Le cycle vital de Parvatrema isostoma Belopolskaja, ig66 (Trematoda : Gymnophallidae) (en Russe). Dokl. Akad. Nauk. SSSR, 1968, I8 3, 1229-1231.

James B. L. : The life cycle of Parvatrema homoeotecnum sp. nov. (Trematoda : Digenea) and a review of the family Gymnophallidae Morozov, I955. Parasitology, GB, 1964, 54 , I-4I.

Loos-Frank, B. : Zur kenntnis der Gymnophalliden Trematoden des Nordseeraumes. III. Gymnophallus gibberosus n. sp. und seine Metacercarie. Z. Parasitenk., I971, 35, 270-281.

REBECQ J. : Recherches systématiques, biologiques et écologiques sur les formes larvaires de quelques Trématodes de Camargue. Thèse Université Aix-Marseille, 1964, n ${ }^{\circ} \mathrm{NRS}$ AO I22, $254 \mathrm{p}$.

Sannia A. : Digenea from marine molluscs. Ph. D. Thesis, I977, University College of Swansea, University of Wales. 